\title{
Simple and reliable fabrication of bioinspired mushroom-shaped micropillars with precisely controlled tip geometries
}

\author{
Hoon Yi, ${ }^{\dagger}$, Minsu Kang, ${ }^{\dagger}$ Moon Kyu Kwak ${ }^{*}$ and Hoon Eui Jeong, ${ }^{*}{ }^{\dagger}$ \\ ${ }^{\dagger}$ Department of Mechanical Engineering, Ulsan National Institute of Science and Technology, Ulsan 689-798, \\ Republic of Korea \\ * Department of Mechanical Engineering, Kyungpook National University, Daegu 702-701, Republic of Korea
}

*Corresponding authors: Email: hoonejeong@unist.ac.kr 


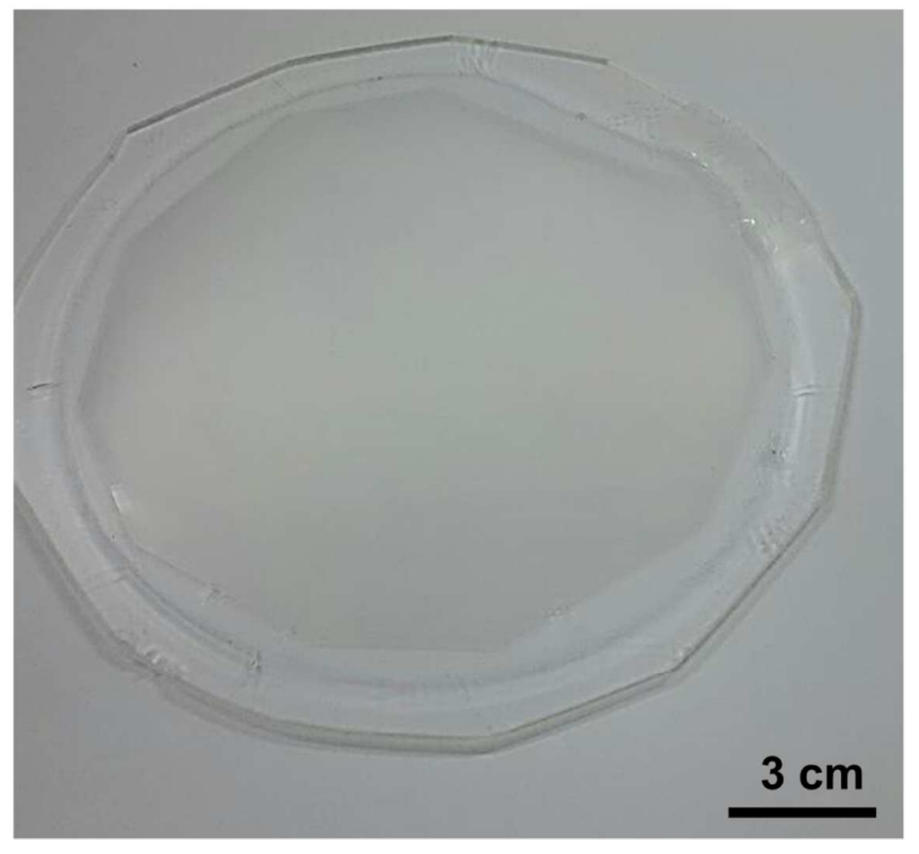

Figure S1 Photograph showing the fabricated large area (6 inch) dry adhesive with mushroom-shaped micropillar arrays. 
a

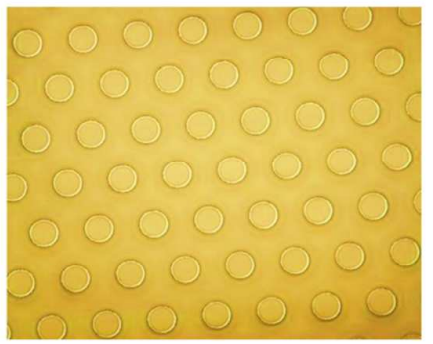

d

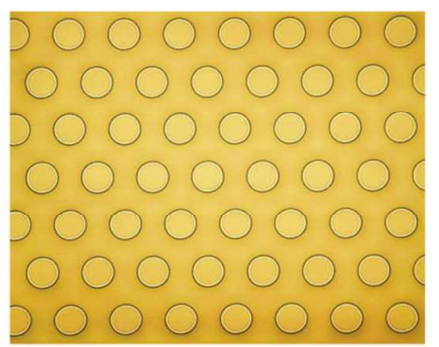

g

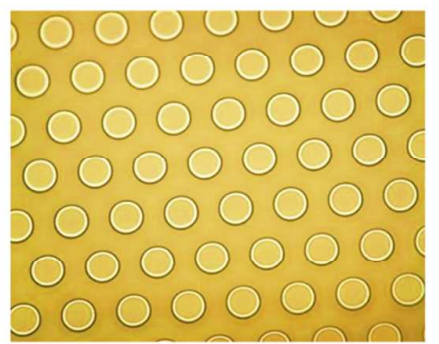

b

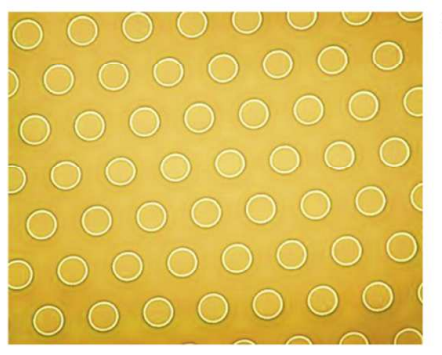

e

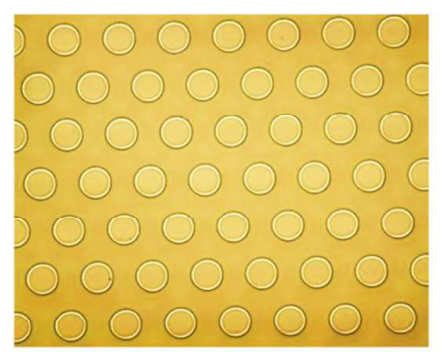

h

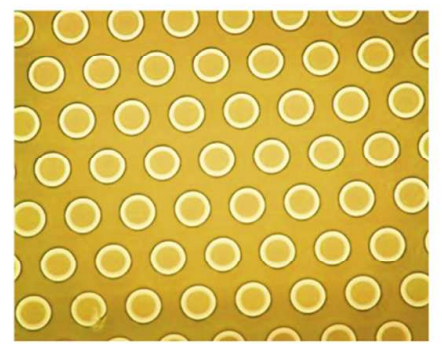

c

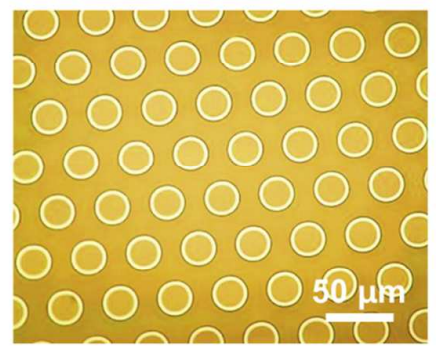

f

D) 000000

(1) 00000

D. 000000

( ) 000000

DOOOOOOO

(1) 00000

DOO 0000

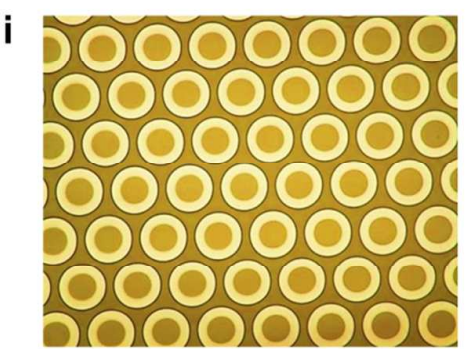

Figure S2 Optical microscopy images of the mushroom-shaped micropillars with different tip diameters and tip thicknesses replicated from the Si masters. (a) $2.10 \mu \mathrm{m} / 20.1 \mu \mathrm{m}$ (tip thickness / tip diameter), (b) $2.10 \mu \mathrm{m} / 22.3 \mu \mathrm{m}$, (c) $2.10 \mu \mathrm{m} / 24.4 \mu \mathrm{m}$, (d) $2.75 \mu \mathrm{m} / 20.6 \mu \mathrm{m}$, (e) $2.75 \mu \mathrm{m} / 22.4 \mu \mathrm{m}$, (f) $2.75 \mu \mathrm{m} / 29.5 \mu \mathrm{m}$, (g) $4.05 \mu \mathrm{m} / 21.6 \mu \mathrm{m}$, (h) $4.05 \mu \mathrm{m} / 24.8 \mu \mathrm{m}$ and (i) $4.05 \mu \mathrm{m} / 33.6 \mu \mathrm{m}$. 

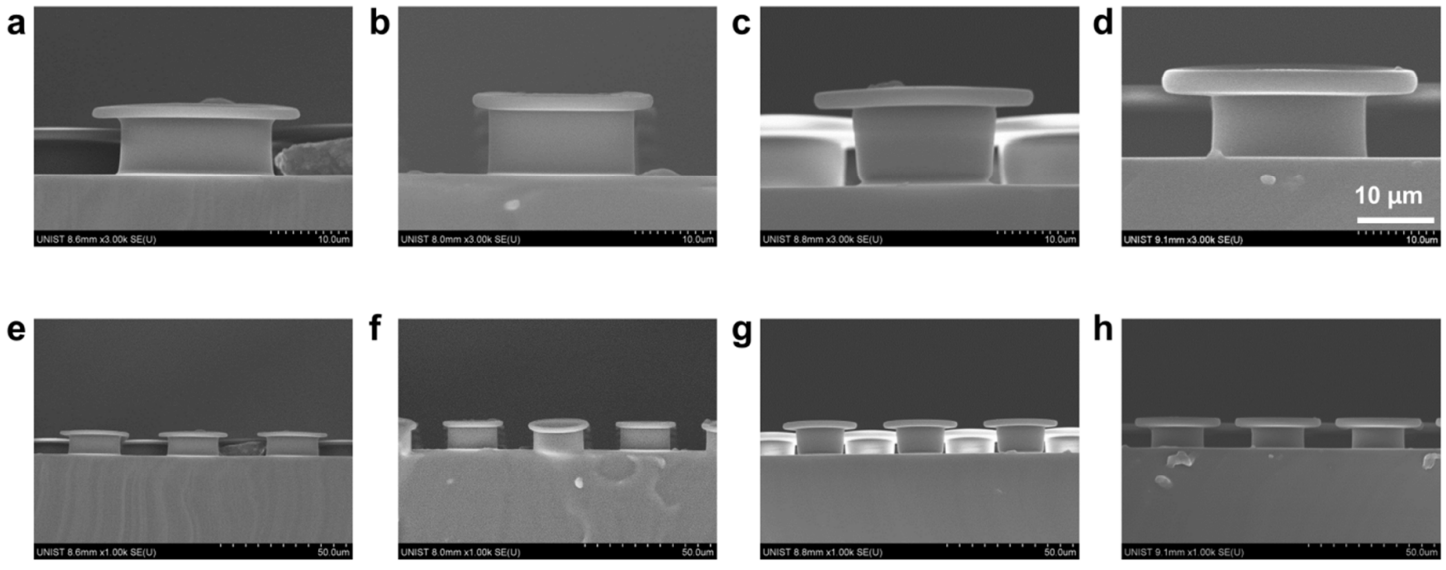

Figure S3 Cross-sectional scanning electron microscopy images of the fabricated micropillars. Micropillars (a, e) with $1.15 \mu \mathrm{m}$ tip thickness and $28.0 \mu \mathrm{m}$ tip diameter, (b, f) with $2.10 \mu \mathrm{m}$ tip thickness and $24.4 \mu \mathrm{m}$ tip diameter, (c, g) with $2.75 \mu \mathrm{m}$ tip thickness and $29.5 \mu \mathrm{m}$ tip diameter, and $(\mathrm{d}, \mathrm{h})$ with $4.05 \mu \mathrm{m}$ tip thickness and $33.6 \mu \mathrm{m}$ tip diameter. 


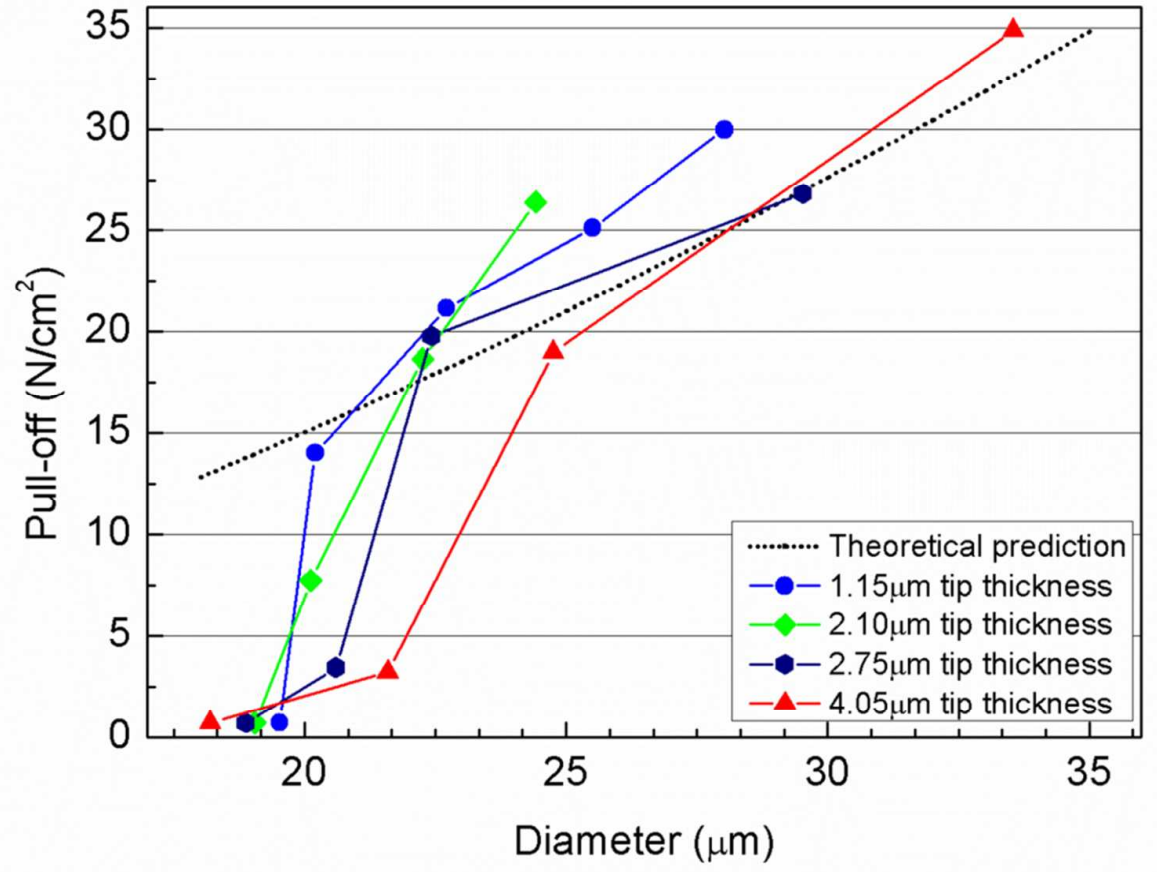

Figure S4 Measurements of the pull-off strength of mushroom-like micropillars having different tip diameters for four different tip thicknesses together with the theoretical predictions. 

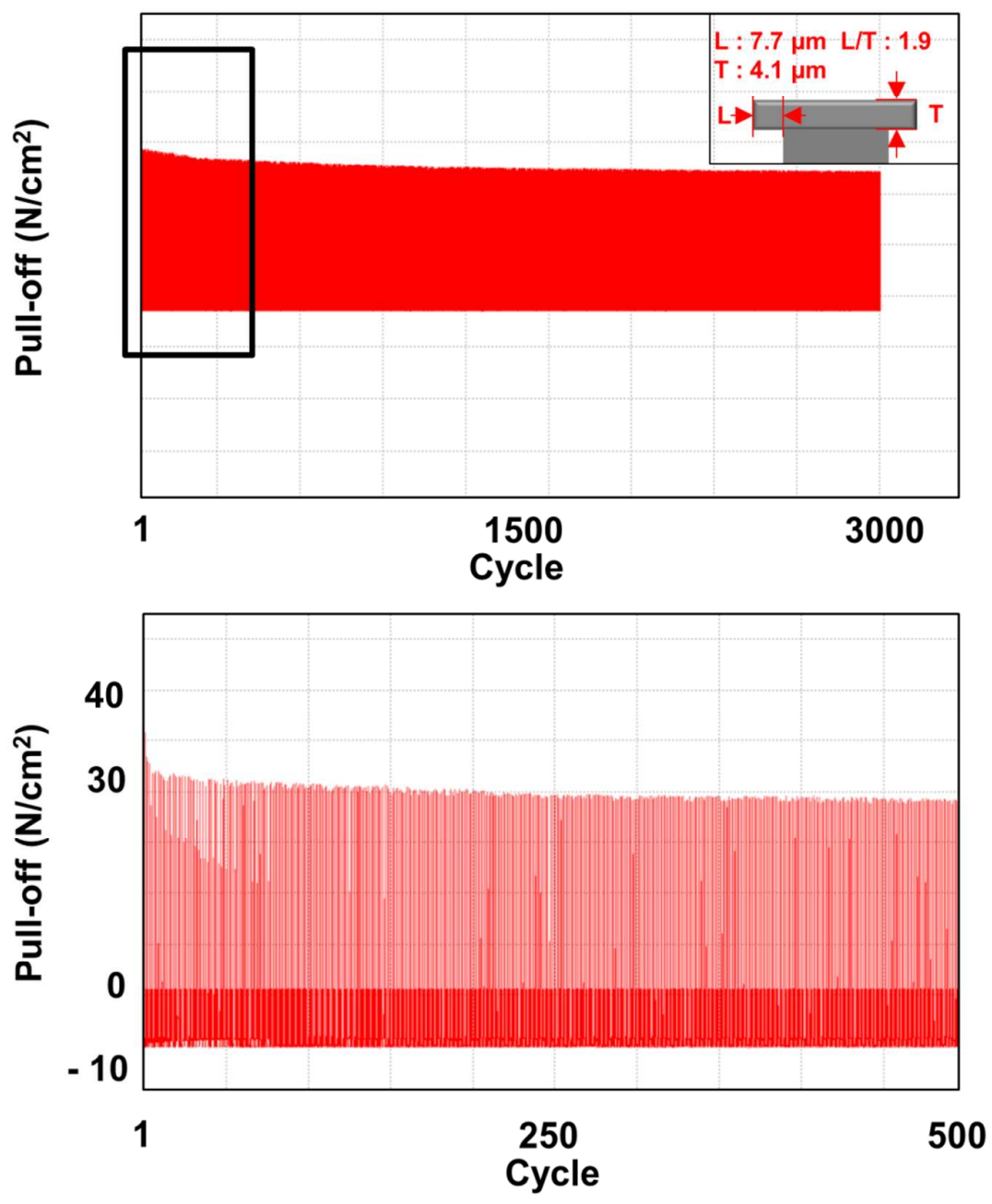

Figure S5 Durability test of the fabricated mushroom-like micropillars with $4.05 \mu \mathrm{m}$ tip thickness and $33.6 \mu \mathrm{m}$ tip diameter. 
Table S1 Comparisons of adhesion performances of mushroom-shaped microstructures with different geometries.

\begin{tabular}{|c|c|c|c|c|c|c|c|c|}
\hline Material & $\begin{array}{l}\text { Pillar diameter } \\
(\mu \mathrm{m})\end{array}$ & $\begin{array}{l}\text { Tip diameter } \\
(\mu \mathrm{m})\end{array}$ & $\begin{array}{l}\text { Tip thickness } \\
(\mu \mathrm{m})\end{array}$ & $\begin{array}{l}\text { Total height } \\
(\mu \mathrm{m})\end{array}$ & $\begin{array}{l}\text { Measurement } \\
\text { probe }\end{array}$ & Preload & $\begin{array}{l}\text { Max. Pull- } \\
\text { off }\end{array}$ & Ref. \\
\hline PDMS & 20 & $20-40$ & 1.5 & 20 & Flat silicon & $4 \mathrm{~N} / \mathrm{cm}^{2}$ & $8.6 \mathrm{~N} / \mathrm{cm}^{2}$ & 1 \\
\hline $\begin{array}{l}\text { PDMS, } \\
\text { Polyurethane }\end{array}$ & $10-76$ & $13-80$ & 1.5 & $12.6-26$ & $\begin{array}{l}1-6 \mathrm{~mm} \text { diameter } \\
\text { sapphire indenter }\end{array}$ & $60 \mathrm{mN}$ & $65 \mathrm{mN}$ & 2 \\
\hline PDMS & $7-9$ & $7-25$ & $2-3$ & 18 & Flat silicon & $0.8 \mathrm{~N} / \mathrm{cm}^{2}$ & $2.7 \mathrm{~N} / \mathrm{cm}^{2}$ & 3 \\
\hline PDMS & 10 & $11.5-16.4$ & 1.5 & 20 & $\begin{array}{l}6 \mathrm{~mm} \text { diameter } \\
\text { sapphire } \\
\text { hemisphere }\end{array}$ & $60 \mathrm{mN}$ & $42 \mathrm{~N} / \mathrm{cm}^{2}$ & 4 \\
\hline PDMS & 10,25 & $12.9,32.1$ & N/A & $\sim 20$ & $\begin{array}{l}5 \mathrm{~mm} \text { diameter } \\
\text { sapphire } \\
\text { hemisphere }\end{array}$ & $1 \mathrm{mN}$ & $17 \mathrm{~N} / \mathrm{cm}^{2}$ & 5 \\
\hline $\begin{array}{l}\text { Polyimide, } \\
\text { PDMS }\end{array}$ & $3.2-20$ & $7-40$ & 1,3 & $20-100$ & Flat glass & $\mathrm{N} / \mathrm{A}$ & $21.9 \mathrm{~N} / \mathrm{cm}^{2}$ & 6 \\
\hline PDMS & 15,20 & 17,22 & $\mathrm{~N} / \mathrm{A}$ & 20 & Flat glass & $5.19 \mathrm{~N} / \mathrm{cm}^{2}$ & $10.4 \mathrm{~N} / \mathrm{cm}^{2}$ & 7 \\
\hline PVS & 25 & 40 & 2 & 100 & Flat glass & $1.3 \mathrm{~N} / \mathrm{cm}^{2}$ & $6 \mathrm{~N} / \mathrm{cm}^{2}$ & 8 \\
\hline Polyurethane & $90.1-66.7$ & 100 & $\begin{array}{l}\text { Tip wedge } \\
\text { angle } 30-60^{\circ}\end{array}$ & 100 & $\begin{array}{l}6 \mathrm{~mm} \text { diameter } \\
\text { glass indenter }\end{array}$ & $2.5 \mathrm{mN}$ & $3.7 \mathrm{mN}$ & 9 \\
\hline Polyurethane & 4.5 & 9 & $\mathrm{~N} / \mathrm{A}$ & 20 & Glass hemisphere & $12 \mathrm{~N} / \mathrm{cm}^{2}$ & $18 \mathrm{~N} / \mathrm{cm}^{2}$ & 10 \\
\hline $\begin{array}{l}\text { Polyurethane } \\
\text { acrylate/PDMS } \\
\text { composite }\end{array}$ & 5 & 7 & N/A & 15 & Flat glass & $0.1 \mathrm{~N} / \mathrm{cm}^{2}$ & $11.4 \mathrm{~N} / \mathrm{cm}^{2}$ & 11 \\
\hline
\end{tabular}




\begin{tabular}{|c|c|c|c|c|c|c|c|c|}
\hline $\begin{array}{l}\text { Polyurethane } \\
\text { acrylate }\end{array}$ & 15,20 & 17,22 & N/A & 20 & Flat glass & $103.9 \mathrm{~N} / \mathrm{cm}^{2}$ & $38.7 \mathrm{~N} / \mathrm{cm}^{2}$ & 12 \\
\hline PMMA & $5.4-15$ & $11.3-14.4$ & N/A & $22-47.8$ & Flat silicon & $3.5 \mathrm{~N} / \mathrm{cm}^{2}$ & $\begin{array}{l}0.175 \\
\mathrm{~N} / \mathrm{cm}^{2}\end{array}$ & 13 \\
\hline
\end{tabular}




\section{References}

1. Sameoto, D.; Menon, C. A Low-Cost, High-Yield Fabrication Method for Producing Optimized Biomimetic Dry Adhesives. J. Micromech. Microeng. 2009, 19, 115002-115008.

2. $\quad$ Sameoto, D.; Ferguson, B. Robust Large-Area Synthetic Dry Adhesives. J. Adhes. Sci. Technol. 2014, 28, $337-353$.

3. Wang, Y.; Shao, J. Y.; Ding, Y. C.; Li, X. M.; Tian, H. M.; Hu, H. Effects of Contact Cap Dimension on Dry Adhesion of Bioinspired Mushroom-Shaped Surfaces. Proc Spie 2015, 9429, 94291E.

4. Sameoto, D.; Sharif, H.; Menon, C. Investigation of Low-Pressure Adhesion Performance of Mushroom Shaped Biomimetic Dry Adhesives. J. Adhes. Sci. Technol. 2012, 26, 2641-2652.

5. del Campo, A.; Greiner, C.; Arzt, E. Contact Shape Controls Adhesion of Bioinspired Fibrillar Surfaces. Langmuir 2007, 23, 1023510243.

6. Davies, J.; Haq, S.; Hawke, T.; Sargent, J. P. A Practical Approach to the Development of a Synthetic Gecko Tape. Int. J. Adhes. Adhes. 2009, 29, 380-390.

7. Y Yi, H.; Hwang, I.; Sung, M.; Lee, D.; Kim, J. H.; Kang, S. M.; Bae, W. G.; Jeong, H. E. Bio-Inspired Adhesive Systems for NextGeneration Green Manufacturing. Int. J. Precis. Eng. Manuf.-Green Tech 2014, 1, 347-351.

8. Gorb, S.; Varenberg, M.; Peressadko, A.; Tuma, J. Biomimetic Mushroom-Shaped Fibrillar Adhesive Microstructure. J. R. Soc. Interface 2007, 4, 271-275.

9. Marvi, H.; Song, S.; Sitti, M. Experimental Investigation of Optimal Adhesion of Mushroom Like Elastomer Microfibrillar Adhesives. Langmuir 2015, 31, 10119-10124.

10. Kim, S.; Sitti, M. Biologically Inspired Polymer Microfibers with Spatulate Tips as Repeatable Fibrillar Adhesives. Appl. Phys. Lett. 2006, 89, 261911.

11. Bae, W. G.; Kwak, M. K.; Jeong, H. E.; Pang, C.; Jeong, H.; Suh, K. Y. Fabrication and Analysis of Enforced Dry Adhesives with Core-Shell Micropillars. Soft Matter 2013, 9, 1422-1427.

12. Yi, H.; Hwang, I.; Lee, J. H.; Lee, D.; Lim, H.; Tahk, D.; Sung, M.; Bae, W. G.; Choi, S. J.; Kwak, M. K.; Jeong, H. E. Continuous and Scalable Fabrication of Bioinspired Dry Adhesives Via a Roll-to-Roll Process with Modulated Ultraviolet-Curable Resin. ACS Appl. Mater. Interfaces 2014, 6, 14590-14599.

13. Hu, H.; Tian, H. M.; Li, X. M.; Shao, J. Y.; Ding, Y. C.; Liu, H. Z.; An, N. L. Biomimetic Mushroom-Shaped Microfibers for Dry Adhesives by Electrically Induced Polymer Deformation. ACS Appl. Mater. Interfaces 2014, 6, 14167-14173. 
